Aus der Prosektur des Krankenhauses München r. I.

(Prosektor: Priv.-Doz. Dr. Oberndorfer.)

\title{
Ueber Beziehungen zwischen Tabes und Leptomeningitis.
}

Von Dr. M. Astwazaturow in St. Petersburg.

Die Leptomeningitis ist, wie bekannt, eine sehr häufige Begleiterscheinung der anatomischen Veränderungen bei Tabes dorsalis. Es wird sogar von manchen Autoren angenommen, daß die Kombination der Tabes mit Meningitis nicht nur ein häufiger, sondern regelmäßiger Befund ist und daß Tabes und Meningitis in kausaler Beziehung zueinander stehen in dem Sinne, daß die meningitische Veränderung das Primum movens der tabischen Degeneration bildet.

Nach Obersteiner und Redlich ${ }^{1}$ ) liegt der Ausgangspunkt der Tabes in der Finschnürung der hinteren Wurzeln, welche durch die meningitische Verdickung und Schrumpfung der Pia zustande kommt.

Auch Nageotte ${ }^{2}$ ) nimmt an, daß die Tabes nichts anderes als die Folge der Wurzelneuritis sei (,Neurite radiculaire interstitielle transverse") und daß diese Neuritis durch luetischmeningitische Veränderungen hervorgerufen wird. Nach der Ansicht des Autors ist die Lymphozytose, die ja in allen Fällen von Tabes sehr früh zur Beobachtung kommt, der beste Beweis der meningitischen Natur dieser Krankheit. Er betrachtet die Tabes als eine der progressiven Paralyse ganz analoge Erkrankung (Meningoencephalitis-Meningomyelitis).

Demgegenüber sind andere Autoren der Ansicht, daß die Kombination der Tabes mit Meningitis zwar häufig, doch nicht regelmäßig, und daß schon deshalb die meningitische Theorie der Tabes nicht anzunehmen sei, abgesehen von einer ganzen Reihe anderer Tatsachen, die gegen diese Theorie sprechen. (Schmaus ${ }^{3}$ ), Oppenheim ${ }^{4}$, Schwarz ${ }^{5}$ ) u. a.)

Ohne die meningitische Theorie der Tabes anzunebmen, sieht $\left.\mathrm{Er}^{6}\right)$ in den Fällen der mit Meningitis verlaufenden Tabes einen weiteren Beweis der luetischen Natur der Krankheit. Diese Fälle können nach ihm als Uebergänge zwischen spezifischer und metasyphilitischer Erkrankung betrachtet werden.

Außer den oben angeführten Fällen von echter Tabes, die mit mehr oder weniger ausgesprochener Meningitis verlaufen, kann die Beziehung zwischen Tabes und Meningitis in umgekehrter Richtung zutage treten, indem die echte Meningitis luetica sekundär die Veränderungen der hinteren Stränge hervorruft und mit tabischen Erscheinungen verläuft. Solche Fälle sind von mehreren Autoren beschrieben worden, und Oppenheim ${ }^{7}$ ) hat für sie die Bezeichnung „Pseudotabes syphilitica" vorgeschlagen.

Diese Fälle von "Pseudotabes syphilitica" sind selbstverständlich als ein sehr günstiges Objekt für die antiluetische Behandlung zu betrachten. Es ist sehr wahrscheinlich, daß gerade diese Fälle zur Meinung geführt haben, daß die antiluetjsche Behandlung der Tabes dorsalis zweckınäßig sei. Daß aber diese Meinung nicht allgemein richtig ist, geht schon daraus hervor, daß neben ihr eine ganz entgegengesetzte und

1) Red lich, Die Pathologie der tabischen Hinterstrangserkrankung. $\left.{ }^{2}\right)$ Nageotte, Progrès méd. 1900. - ") Schmaus, Vorlesungen ïber die pathologische Anatomie des Rückenmarks 1901, S. 179. $\left.{ }^{4}\right)$ Oppenheim, Lehrbuch der Nervenkrankheiten. 5. Auflage. 5) Sehwarz, Zeitschrift für Heilkunde, Bd. 18, H. 2 u. 3. -6) Erb, Deutsche Zeitschrift für Nervenkrankheiten, Bd. 22. - ${ }^{7}$ ) Oppen
heim, Lehrbuch der Nervenkrankheiten, 5. Auflage, Bd. 1, S. 353. 
mehr verbreitete Anschauung existiert, nämlich, daß die antiJuetische Behandlung der Tabes nicht nur zwecklos, sondern sogar schädlich ist. "Ich habe von derselben" - sagt Prof. Oppenheim - ",vorwiegend Mißerfolge zu verzeichnen."

Obwohl aber im allgemeinen die Meinung über den Nutzen der antiluetischen Behandlung bei Tabes dorsalis nicht zutreffend ist, muß es doch Fälle geben, für welche diese Meinung zu Recht besteht. Das sind wahrscheinlich Fälle von „Pseudotabes syphilitica" oder Tabesfälle, die mit spezifisch luetischen Veränderungen kombiniert sind.

Vielleicht darf diese Tatsache besonders jetzt betont werden, da wir im Salvarsan allem Anschein nach ein Mittel an die Hand bekommen haben, das sich auch gegen luetische Veränderungen des Zentralnervensystems wirksam zeigt.

Jedenfalls scheint es wichtig zu sein, die klinischen Merkmale festzustellen, die für Pseudotabes syphilitica resp. fïr deren Unterscheidung von der echten Tabes charakteristisch sind.

In dieser Beziehung ist der folgende Fall nicht ohne Interesse.

Der betreffende Patient wurde seit mohr als 20 Jaliren für einen Tabiker gehalten. Dabei waren die klinischen Erscheinungen zwar etwas eigenartig, doch stimmten sie im allgemeinen mit der Diagnose der Tabes vollkommen überein. Anatomisch aber erwies sich dieser Fall als „Pseudotabes syphilitica“. Die meningitischen Erscheinungen waren hicr so hervorstechend, daß es nahelicgt, gerade diesen Veränderungen die Eigenartigkeit des klinischen Verlaufes zuzuschreiben.

Die wichtigsten anamnestischen und klinischen Angaben, dic ich Herrn Hofrat Dr. Hoferer in München verdanke, sind folgende: Der Kranke war seines Standes Kaufmann. Luesinfektion unbestimmt in welchem Jahre. Konsultation des Prof. Kussmaul in Straßburg wegen "des Magens". Es traten zeitweisc Brechanfälle mit heftigen Magenschmerzen auf. Prof. Kussniaul führte diese Brechanfälle als „Crises gastriques" auf ein Rückenmarkleiden zurück und soll außerdem bereits Fehlen der Patellarsehnenreffexe sowie reflektorische Pupillenstarre nachgewiesen haben.

Kranklieitsgeschichte ab 1890. Inl April 1890 ein heftiger Anfall von "Crises gastriques", der drei Wochen lang dauerte, verbunden mit häufigem Erbrechen und Magenschmerzen. Ein gleich heftiger Anfall inı September 1890. Blasenschwäche (Parese des M. detrusor); Katheterismus. Die Blasensehwäche bestand weiterhin bis zum Tode un. unterbrochen fort, sodaß Katheterismus sich als notwendig erwies. Dic von Kussniaul bercits nachgewiesenen charakteristischen Haupterscheinunger (Fehlen der Patellarsehnenreflexe sowie reflektorische Pupillenstarre) bestanden andaucrnd weiter; es gesellten sich sogar noch Lähuunnen der Augenmuskeln im Gebiete des N. oculomotorius hinzu. Ataktische Störungen von seiten der Füße oder Arınc traten nic auf; hingegen bildete eine der Hauptklagen in dem langdaucrnden Krankheitsverlaufe einc sehr starke Hyperästhesic der Haut, besonders am Abdomen, an Armen und Füßen, sowie die heftigsten lanzinierenden, blitzartigen Schmerzen in den Armen und Beinell. Diese Schmerzen waren derart heftig, daß der Kranke lant aufschrie. Beim Nachlassen dicser lanzinierenden Schmerzen trat als Folge gewöhnlich ein äußerst lästiger Druck in der Magengegend, ausstrahlend nach dem Rücken auf. Die ständige Klagc von seiten des Kranken lautete dann: „Herr Doktor, mein Magen, mein Magen!" Dabei war dic Funktion des Magens normal, und es konnte reichliche Nahrung ohne besondere Auswahl aufgenommen werden. Gewöhnlich schlossen sich dem Magendruck Brechanfälle an, die in den letzten Jalıren der Krankhcit schwächer wurden und schließlich ganz aufhörten.

Inı ganzen Krankheitsverlaufe war ein ganz bestimmter Zyklus im Ablauf der Schmerzen und Beschwerden eliarakteristisch, insofern, als den Crises gastriques die lanzinierenden Schmerzen, diesen der Magendruck etc in einer ganz bestinımten Regelmäßigkeit und zeit. lichen Aufeinanderfolge folgten. In den letzten Jahren traten vorübergehend Oedeme umschrieben an eincul F'ußknöchel auf. Ferner Herzkrisen, welche in tachy- und bradykardischem Charakter sich abwechselnd äußerten. Auch Gehirnkrisen vorübergehender Natur nit Scliwindel, Sprachstörungen, Trübung des Bewußtseins konnten lin und wieder beobachtet werden.

Anı 6 April 1909 trat eine akute fibrinöse Bronchitis auf, die bis 15. April daucrte. Am 7. Juni trat Fieber auf in Begleitung von Magellschmerzen und starker Trübung des Bewußtseins, Cheyne-Stokessche Atmung. Anı 19. Juni Exitus letalis. Die Sektion (Dr. Obern (łorfer) zeigte ausgesprochene Aufhellung der Hinterstränge des Rückenmarks, daneben eine enorme Verdickung der weichen Häute nit ausgedelinten Adliäsionen mit der Dura in der ganzen Ausdelinung des Markes.

Bei der mikroskopisclien Untersuchung schien die Pia außerordentliclı verdickt und selır stark mit Rundzellen infiltriert (Fig. 1).
Diese rundzellige Infiltration war auch in dem Gebicte der hinteren und vorderen Wurzeln zu konstatieren. Besonders reich war sie abcr in der Umgebung der Piagefäße; sie begleitete auch die bindegewebigen Ausläufer, welche von der Pia in die Rückenmarksubstanz hineindringen und welche in unserem Falle eine merkliche Verdickung zeigten.

Außer der Infiltration fiel eine reiche Bindegewebswucherung sowohl in der Pia als in den Wurzeln auf.

Bei der speziellen Färbung der Nervenfasern konnte man sieh überzeugen, daß von den hinteren Wurzeln fast nichts geblieben war und daß sie sich mit bindegewebigen Fasern vollkommen durchsetzt zeigten (Fig. 2).

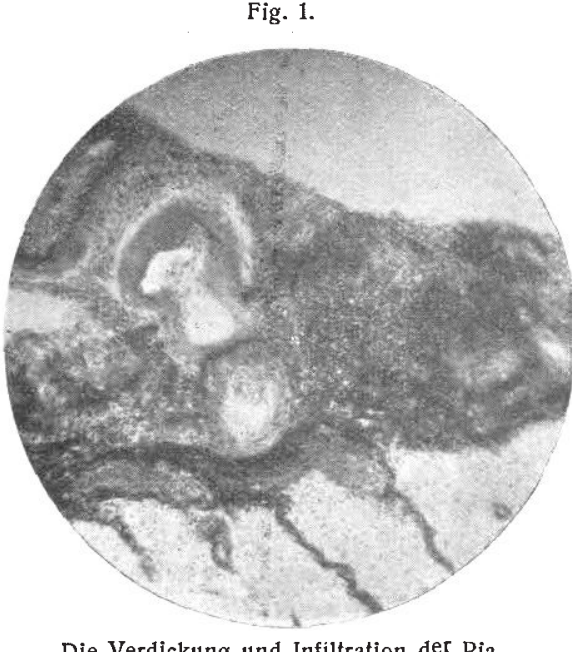

Die Verdickung und Infiltration der Pia. Van Giesonsche Färbung.
Im allgemeinen scheinen die entzündlichen Erscheinungen in den hinteren Teilen zu prävalieren, sodaß das Bild mehr der „Meningitis posterior" entspricht. Jedoch fehlen die meningitischen Erscheinungen

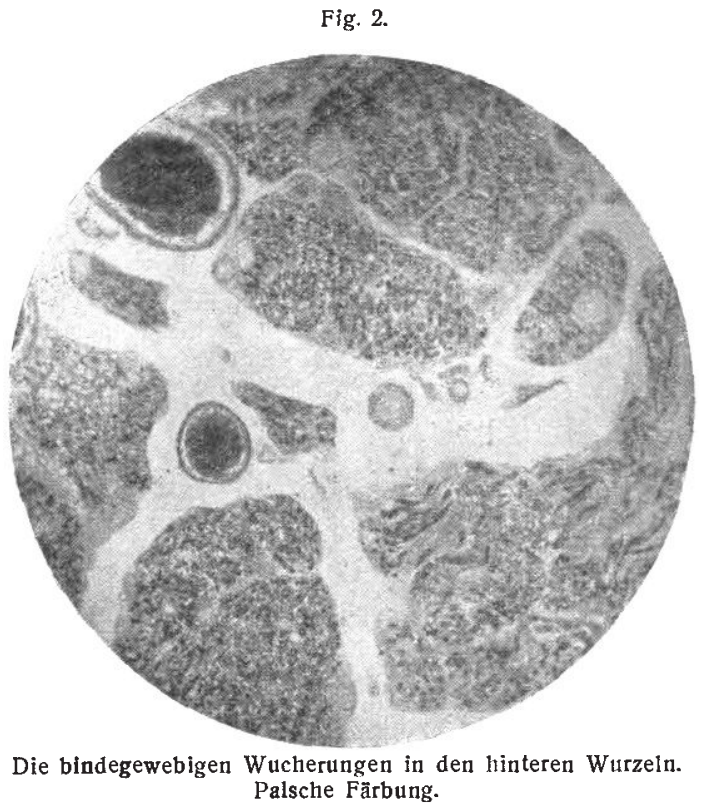

auch in vorderen Teilen nicht. Auch sind, wie erwälınt, einzehne rundzellige Herde im Rückenmark sclbst zu konstatieren, sodaß die Diagnose der Meningomyelitis nicht unberechtigt wäre. In allen Höhen des Rückenmarks sind die beschriebenen meningitischen Veränderungen ziemlich gleichmäßig ausgesprochen. Dic Gefäße der Pia und des Rücken. marks zeigen starke sklerotische Veränderung mit Intimaverdickung und mit sehr starker, teilweise zur völligen obliteration führender hyalinen Degeneration.

Was dic tabischen Veränderungen betrifft, so waren die hinteren Stränge in allen Höhen des Rückenmarks degeneriert. Es muß aber bemerkt werden, daß diese Degeneration für die Tabes nicht vollkommen typisch war. Erstens waren außcr in den hinteren Strängen auch in andcren Teilen, wenn auch nicht so starke, Degenerationszeichen zil konstatieren. Ferner war die Degeneration der hinteren Stränge für die Dauer der Krankheit und Intensität der klinischen Symptome nicht cntsprechend stark entwickelt. Besonders in Lumbalteil war die Degeneration wenig ausgeprägt, was bei Tabes ziemlich selten ist. Es fehlte das für Tabes so charakteristische Ueberwiegen der Degeneration in bestimmten Teilen der hinteren Stränge, auch war die Verteilung der Degeneration eine der tabischen entgegengesetzte. So war z. B. im Halstcil die Degeneration nicht, wie das in typischen Fällen von 'Tabes der Fall ist, am stärksten in dem Fasciculus Golli ausgesprochen, sondern die medialen Teile der hinteren Stränge waren ziemlich intakt, am stärksten aber waren die dem Kommafeld entsprcclienden Bahnen an der Degeneration beteiligt, und zwar auf der einen Seite mehr als auf der anderen.

Die angeführten anatomischen Erscheinungen zeigen, daß es sich in unserem Falle kaum um eine echte Tabes ge- 
handelt hat. Man nuß ihn vielmehr als ,Pseudotabes syphilitica" bezeichnen in dem Sinne, daß, obwohl die Entwicklung und die Symptome der Krankheit denjenigen der Tabes entsprachen, sie doch durch meningo-myelitische Veränderungen verursacht waren; oder wenigstens muB man annehmen, daß es sich um einen Fall von Tabes handelte, der mit meningitischen Veränderungen kombiniert war.

Wenn. wir nun die klinischen. Erscheinungen unseres Falles in Betracht ziehen und berïcksichtigen., was in dem Verlauf der Krankheit besonders charakteristisch war, so fallen uns in erster Linie die Schnerzen und Krisen auf. Und es liegt nahe, diese Besonderheit des Verlaufs mit den anatomischen Besonderheiten des Falles in Zusanımenhang zu bringen. Dje Periodizität im Auftreten genannter Erscheinungen findet eine genügende Erklärung in der Tatsache, daß die anatomischen Veränderungen in den. Meningen sehr verschiedenen. Alters waren, indem neben alten, bindegewebigen. Wucherungen ganz frische Infiltrationsherde zu finden waren. Bei der gleichmäßigen. Verbreitung der Meningitis über die ganze Iänge des Rückenmarks ist auch die Multiplizität und dic Ausbreitung der Schmerzen und Krisen verständlich.

Schluß. Der beschriebene Fall zeigt also, daß die ,tabischen. Krisen" meningitischer resp. spezifischer Natur sein können und daß die große Intensität und Ausbreitung der Krisen und Schmerzen bei der Tabes dorsalis den Verdacht einer Pseudotabes syphilitica oder mit Meningitis kolubinierter Tabes rechtfertigen kömnen. Damit stimnen auch manche andere Tatsachen ïberein. Erstens die, daß die krisenartigen und lanzinierenden Schmerzen bei Meningitis keine seltene Erscheinung sind. Zweitens scheinen gerade diese Symptome der Tahes von der antiluetischen Behandlung beeinflußbar zu sein. Nach Angabe von Wechselmann ${ }^{1}$ ) stehen unter den tabischen. Symptomen, welche der Behandlung nit Salvarsan. zugänglich sind, in erster Linie, das Gürtelgefühl, der dumpfe, jahrelang dauernde Kopfschmerz, furchtbar schwere Interkostalneuralgien".

Nach der herrschenden Meinung werden die Krisen durch Sympathikusdegeneration hervorgeruferl. Es ist aber nicht ausgeschlossen und nach den oben angeführten. Gründen. sogar sehr wahrscheinlich, daß in den mit Meningitis verlaufenden. Fällen von Tabes die krisenartigen. Anfälle durch die entzïndlichen Prozesse in den hinteren Wurzeln bedingt werden können.

$\mathrm{Ob}$ nun. der in unserem Falle beobachtete, mit starken Schnerzen und Krisen einhergehende Verlauf für dic „meningitische Form" der Tabes iiberhaupt charakteristisch ist, das kann nicht mit Sicherheit beantwortet werden. 